\title{
Photoluminescence and Raman studies in swift heavy ion irradiated polycrystalline aluminum oxide
}

\author{
K R NAGABHUSHANA, B N LAKSHMINARASAPPA* and FOURAN SINGH ${ }^{\dagger}$ \\ Department of Physics, Jnanabharathi, Bangalore University, Bangalore 560 056, India \\ ${ }^{\dagger}$ Inter University Accelerator Centre, P.O. Box No. 10502, New Delhi 110 067, India
}

MS received 26 September 2008; revised 4 April 2009

\begin{abstract}
Polycrystalline aluminum oxide is synthesized by combustion technique and XRD studies of the sample revealed the $\alpha$-phase. The synthesized sample is irradiated with $120 \mathrm{MeV}$ swift $\mathrm{Au}^{9+}$ ions for the fluence in the range from $1 \times 10^{11}$ to $1 \times 10^{13}$ ions $\mathrm{cm}^{-2}$. A broad photoluminescence (PL) emission with peak at $\sim 447 \mathrm{~nm}$ and two sharp emissions with peak at $\sim 679$ and $\sim 695 \mathrm{~nm}$ are observed in pristine when sample was excited with $326 \mathrm{~nm}$. However, in the irradiated samples the PL intensity at $\sim 447,679$ and $695 \mathrm{~nm}$ decreases with increase in ion fluence. The $\alpha-\mathrm{Al}_{2} \mathrm{O}_{3}$ gives rise to seven Raman modes with Raman intensity with peaks at $\sim 253,396,417,546,630,842,867 \mathrm{~cm}^{-1}$ observed in pristine. The intensity of these modes decreases with increase in ion fluence. However, the Raman modes observed at lower fluences are found to disappear at higher fluence.
\end{abstract}

Keywords. $\quad \mathrm{Al}_{2} \mathrm{O}_{3}$; swift heavy ions; Raman spectra; photoluminescence; amorphization.

\section{Introduction}

Radiation effect in inorganic insulating materials is a subject matter of investigation due to their scientific and technological importance. The major reason of this interest is the need for a better understanding of material performance in radiation environment of nuclear reactors and accelerators. Also, the incorporation of energetic heavy ions as a processing technique improves material properties (Nagabhushana et al 2006; Song et al 2006). Among the insulators aluminum oxide, also known as alumina, is of high interest due to its superior properties such as very stable structure, good electrical insulation, high strength and chemical inertness etc which provides large potential of applications such as RF windows in fusion reactors, toroidal insulating breaks, diagnostic probes, coatings, abrasives, thermoluminescence dosimeters etc (McKeever et al 1999; Mimani 2000; Ryazanov and Kinoshita 2002). Most of the physical properties of aluminum oxide, in particular, its optical and electrical features are dielectric in nature (Kadleikova et al 2001). Swift heavy ions (SHI) cause intense electronic excitations along the ion trajectory when they pass through material that may result in defect production or amorphization or phase transformation on nanometer scale (Bolse et al 2004; Wang et al 2004). Thus, it is interesting to know the effect of strong electronic excitation by high energy

*Author for correspondence (bnlnarasappa@gmail.com) heavy ions in the surface region of materials. With $120 \mathrm{MeV}$ gold ions for aluminum oxide target in the present studies, Se and Sn are calculated using SRIM 2003 program to be $24.83 \mathrm{keV} \mathrm{nm}^{-1}$ and $0.36 \mathrm{keV} \mathrm{nm}^{-1}$, respectively. Here, the Se is higher than the threshold required for the creation of extended defects. Se is dominant up to $\sim 9.1 \mu \mathrm{m}$ from the surface (www.srim.org).

Raman and photoluminescence (PL) spectroscopy are known to be nondestructive material characterization techniques. Raman spectroscopy provides unique ways of probing surface and structural properties of ion beam modified materials. The PL spectrum is correlated to the surface amorphization due to SHI irradiation. In the present paper we report the effect of $120 \mathrm{MeV}$ swift $\mathrm{Au}^{9+}$ ion irradiation on aluminum oxide through glancing angle X-ray diffraction (GAXRD), Raman and PL spectroscopy technique.

\section{Experimental}

Polycrystalline aluminum oxides were synthesized by combustion technique based on the procedure discussed by Kingsley and Patil (1988). Details of the certificate of guarantee (COG) of chemicals used in synthesis are given elsewhere (Nagabhushana 2008). Where assay 99.95\% and other impurities such as chloride ( $\mathrm{Cl}$ ) $0.0005 \%$, sulphate $\left(\mathrm{SO}_{4}\right) 0.03 \%$ and lead $0.005 \%$ are present in the ingredient chemicals. However, there are some more impurities which may also be present in the ingredients. However, elemental analysis of the synthesized $\mathrm{Al}_{2} \mathrm{O}_{3}$ is 
not carried out. Pellets, $6 \mathrm{~mm}$ diameter and $1 \mathrm{~mm}$ thickness, of $\mathrm{Al}_{2} \mathrm{O}_{3}$ were prepared using a home-made pelletizer and by applying a pressure of $70 \mathrm{MPa}$. One of these samples was used as pristine for comparison. The pellets were irradiated with $120 \mathrm{MeV}$ swift $\mathrm{Au}^{9+}$ ions for various fluences in the range $1 \times 10^{11}$ to $1 \times 10^{13}$ ions $\mathrm{cm}^{-2}$ using 15 UD tandem Pelletron accelerator in materials science beam line at the Inter University Accelerator Centre, New Delhi. The structural characterizations of the pristine and SHI irradiated samples are analysed by GAXRD (Bruker $\mathrm{X}$-ray machine, $\mathrm{CuK} \alpha$ rays) technique at a glancing angle of $1^{\circ}$. Raman spectra were recorded using Renishaw, model 1000 Raman spectrometer. A $785 \mathrm{~nm}$ laser beam of $0.5 \mathrm{~mW}$ from an Ar ion laser is used as excitation source. The micro Raman data was recorded in the range $200-900 \mathrm{~cm}^{-1}$. Luminescence technique is a very sensitive technique to identify trace impurities and point defects, hence PL studies were performed using excitation at $326 \mathrm{~nm}$ light from $\mathrm{He}-\mathrm{Cd}$ laser (KIMMON) and Mechelle900 spectrograph. The PL setup has a cooled CCD array-based detection system. The laser light is made to incident on the sample at $45^{\circ}$ and the luminescence light was collected using a collector assembly attached to the spectrograph through optical fibre for detection and analysis.

\section{Results and discussion}

\subsection{Glancing angle X-ray diffraction}

The GAXRD patterns of as synthesized unirradiated aluminum oxide are given in figure $1(\mathrm{a})$. The diffraction peaks at $25 \cdot 25,35 \cdot 16,37 \cdot 78,43 \cdot 36,52 \cdot 58$ and $57 \cdot 52^{\circ}$ are due to (012), (104), (110), (113), (024) and (116), respectively. The results compared to those of bulk $\alpha$-alumina (JCPDS: 82-1468), were found to belong to rhombohedral crystal system with $R \overline{3} c$ space group. Also, the patterns are observed to be in good agreement with polycrystalline $\mathrm{Al}_{2} \mathrm{O}_{3}$ powders reported by Kingsley and Patil (1988). The GAXRD measurements are carried out on irradiated pellets in order to avoid the effect of pristine part in irradiated part, because X-rays penetrate a few microns in bulk at larger angles. In figure $1, \mathrm{XRD}$ of pristine sample is compared with irradiated one (figure 1b). Careful observation of the XRD of both samples revealed that the intensity of the irradiated sample is lower when compared to that in pristine. The reduction in intensity clearly indicates that the sample starts to amorphize after SHI irradiation at the given fluence. Also, Raman studies clearly indicate the amorphization of the sample. Because, Raman spectroscopy is a very sensitive technique depending on the crystal structure, even a little change in the lattice order leads to change in Raman spectra. Furthermore, alumina being a high radiation resistant material one cannot expect complete amorphization at this fluence.

\subsection{Raman studies}

Raman spectroscopy provides associated vibrational spectrum of molecular spectra within the irradiated scattering volume. Aluminum oxide has several crystallographic phases. The two important phases are water free $\mathrm{Al}_{2} \mathrm{O}_{3}$ viz. alpha alumina $\left(\alpha-\mathrm{Al}_{2} \mathrm{O}_{3}\right)$ and gamma alumina $\left(\gamma-\mathrm{Al}_{2} \mathrm{O}_{3}\right)$. Other phases of $\mathrm{Al}_{2} \mathrm{O}_{3}$ are eta alumina $\left(\eta-\mathrm{Al}_{2} \mathrm{O}_{3}\right)$, theta alumina $\left(\theta-\mathrm{Al}_{2} \mathrm{O}_{3}\right)$ and beta alumina $\left(\beta-\mathrm{Al}_{2} \mathrm{O}_{3}\right)$. Among these $\gamma-\mathrm{Al}_{2} \mathrm{O}_{3}$ and $\eta-\mathrm{Al}_{2} \mathrm{O}_{3}$ does not show any bands in Raman spectrum (Kingsley and Patil 1988). $\alpha-\mathrm{Al}_{2} \mathrm{O}_{3}$, also known as corundum, is shown with space group $R \overline{3} c$. The representation of optical modes in $\alpha-\mathrm{Al}_{2} \mathrm{O}_{3}$ are given by Krishnan (1947)

$$
2 A_{\mathrm{lg}}+2 A_{\mathrm{lu}}+3 A_{2 \mathrm{~g}}+2 A_{2 \mathrm{u}}+5 E_{\mathrm{g}}+4 E_{\mathrm{u}}
$$

Among these $2 A_{1 \mathrm{~g}}+5 E_{\mathrm{g}}$ are Raman active, $2 A_{2 \mathrm{u}}+4 E_{\mathrm{u}}$ are IR active whereas $2 A_{1 \mathrm{u}}+3 A_{2 \mathrm{~g}}$ are neither Raman nor IR active modes. Therefore, $\alpha-\mathrm{Al}_{2} \mathrm{O}_{3}$ gives rise to seven Raman active modes. Figure 2 shows the Raman spectra of (a) pristine and SHI irradiated polycrystalline aluminum oxide. Several Raman modes with peaks at $\sim 253$, $396,417,546,630,842,867 \mathrm{~cm}^{-1}$ are observed in pristine. On the basis of Raman polarization study seven Raman active modes have been assigned by Porto and Krishnan (1967). The Raman modes observed in the present studies are assigned and given in table 1 . The Raman bands with peaks at $396,417,546,630$ and $842,867 \mathrm{~cm}^{-1}$ have been assigned to $E_{\mathrm{g}}$ (external), $A_{1 \mathrm{~g}}, E_{\mathrm{g}}$ (external), $A_{1 \mathrm{~g}}, E_{\mathrm{g}}$ (internal) modes, respectively. The other mode corresponding to $E_{\mathrm{g}}$ (external) at $\sim 432 \mathrm{~cm}^{-1}$ is not observed in the present studies. However, an extra band with peak at $253 \mathrm{~cm}^{-1}$ is observed. This might be due to the fact that, the vibrational spectrum of the crystal is modified even by slight changes in the crystal structure (Kadleikova et al 2001). Further, in case of the SHI irradiated samples the intensities of Raman bands decreases

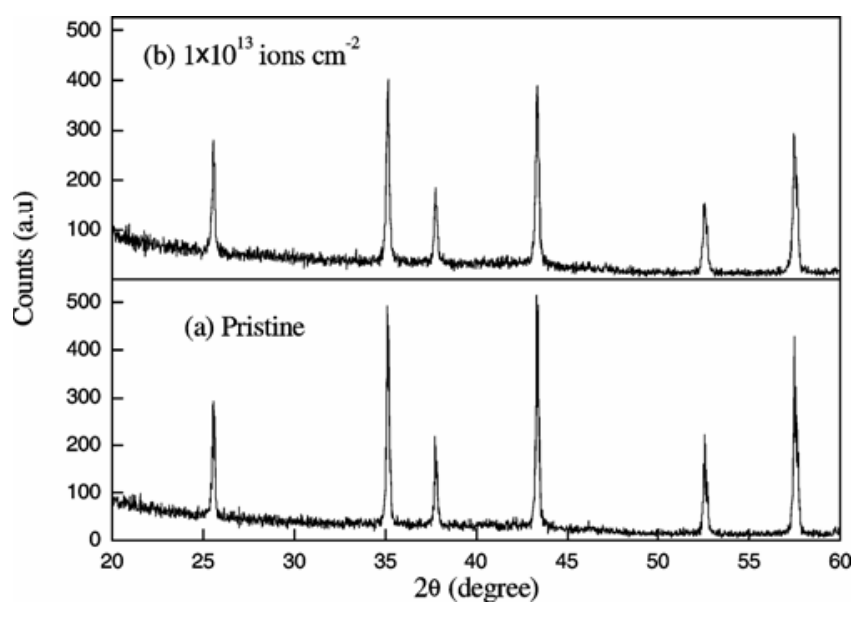

Figure 1. GAXRD patterns of (a) pristine and (b) $120 \mathrm{MeV}$ $\mathrm{Au}^{9+}$ ion irradiated polycrystalline aluminum oxide. 
with increased ion fluence and at the fluence of $1 \times 10^{13}$ ions $\mathrm{cm}^{-2}$ the samples did not show any Raman bands as can be seen from figure 2(d). This might be due to either change in the crystal phase or surface amorphization. However, XRD studies reveal no phase transformation at the fluence of $1 \times 10^{13}$ ions $\mathrm{cm}^{-2}$ as discussed in $\$ 3.1$ above. It is well known that Raman spectroscopy is a powerful tool to evaluate the induced amorphization of a crystalline structure. Raman spectra of crystalline with lattice defects and systems having other types of struc-

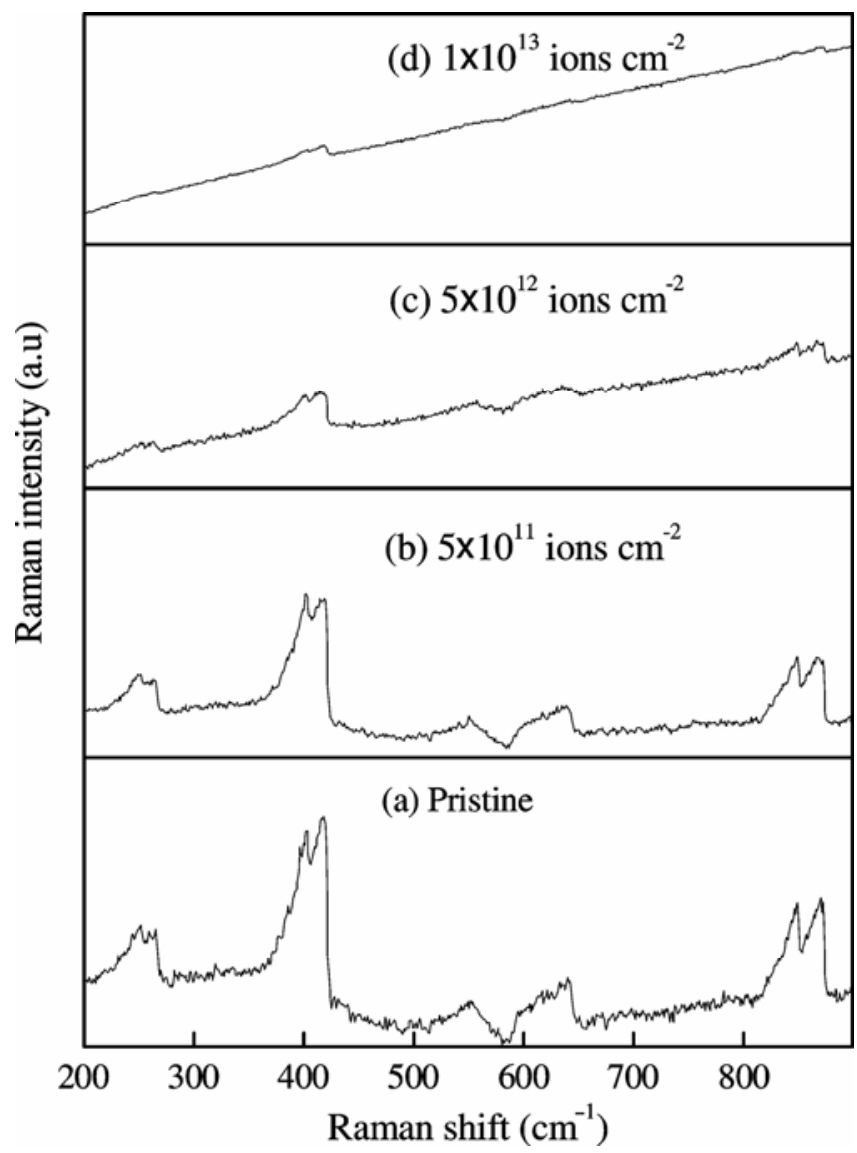

Figure 2. Raman spectra of pristine and $\mathrm{SHI}$ irradiated polycrystalline $\mathrm{Al}_{2} \mathrm{O}_{3}$.

Table 1. Peak positions of Raman modes of pristine and $120 \mathrm{MeV} \mathrm{Au}{ }^{9+}$ ion irradiated combustion synthesized polycrystalline aluminum oxide.

\begin{tabular}{llcc}
\hline $\begin{array}{l}\text { Peak position } \\
\left(\mathrm{cm}^{-1}\right)\end{array}$ & \multicolumn{1}{c}{ Pristine } & $\begin{array}{c}5 \times 10^{11} \\
\text { ions cm }\end{array}$ & $\begin{array}{c}1 \times 10^{13} \\
\text { ions cm }\end{array}$ \\
\hline 253 & No assignment & & - \\
396 & $E_{\mathrm{g}}$ (external) & $E_{\mathrm{g}}($ external) & - \\
417 & $A_{\mathrm{lg}}$ & $A_{\mathrm{lg}}$ & $A_{\mathrm{lg}}$ \\
546 & $E_{\mathrm{g}}($ internal $)$ & $E_{\mathrm{g}}($ internal $)$ & - \\
630 & $A_{\mathrm{lg}}$ & $A_{\mathrm{lg}}$ & - \\
842 & $E_{\mathrm{g}}($ internal $)$ & $E_{\mathrm{g}}$ (internal) & - \\
867 & $E_{\mathrm{g}}($ internal $)$ & $E_{\mathrm{g}}$ (internal) & $E_{\mathrm{g}}$ (internal) \\
\hline
\end{tabular}

tural disorder usually exhibit a pronounced line broadening in comparison with ordered structures (Brundle et al 1992). However, in the present case there is an appreciable line broadening and large decrease in Raman intensity as can be seen from figure 2 . This might be attributed to high defect concentrations causing broadened Raman lines. Further, Raman spectra suggest that there is structural damage to $\mathrm{Al}_{2} \mathrm{O}_{3}$ lattice upon ion irradiation. SRIM results indicate that when the energy of the incident ion is of a few keV then the nuclear energy loss $\left(S_{\mathrm{n}}\right)$ is significant as compared to electronic energy loss $\left(S_{\mathrm{e}}\right)$ (Ziegler 2004). In the present studies, $120 \mathrm{MeV} \mathrm{Au}^{9+}$ ions are used for irradiation and the electronic energy loss is dominant over the nuclear energy loss. The incident ions are expected to break the $\mathrm{Al}-\mathrm{O}$ bonds in $\mathrm{Al}_{2} \mathrm{O}_{3}$. The disordered lattice will contribute to the internal stress and thus the Raman peak intensity decreases. The vibration spectrum of crystal is modified even by slight changes in the crystal structure such as sample of different origin and substitution by different cations and anions and creation of radiation induced defects (Nagabhushana et al 2008).

\subsection{Photoluminescence}

Figure 3 shows PL spectra of pristine and SHI irradiated polycrystalline aluminum oxide. A broad PL emission with peak at $447 \mathrm{~nm}$ in the range $400-600 \mathrm{~nm}$ and two sharp PL emissions with peak at $\sim 679$ and $695 \mathrm{~nm}$ have been observed in all samples. The two sharp emissions are attributed to well known $\mathrm{Cr}^{3+}$ doublet $R_{1}$ and $R_{2}$ lines. Trace quantities of $\mathrm{Cr}^{3+}$ ions are naturally present in ceramic alumina. $\mathrm{Cr}^{3+}$ ions are residing in an octahedrally coordinated oxygen environment. The luminescence due to $\mathrm{Cr}^{3+}$ ions is used as a probe for the crystalline environment because of its high sensitivity to the variations

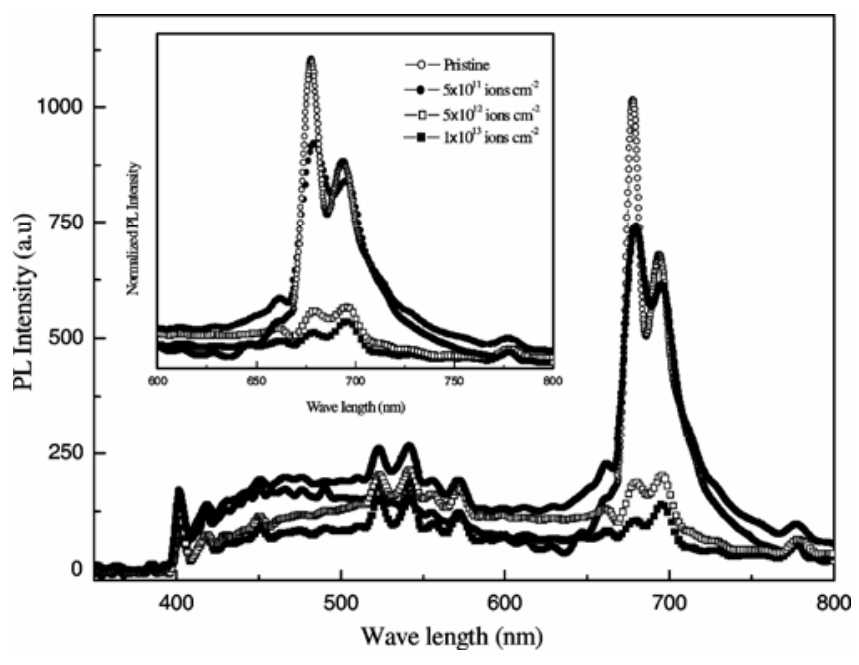

Figure 3. Photoluminescence $\left(\lambda_{\mathrm{ex}}=326 \mathrm{~nm}\right)$ of pristine and $\mathrm{SHI}$ irradiated polycrystalline $\mathrm{Al}_{2} \mathrm{O}_{3}$. 
in crystal field (Jankowiak et al 2000). Makhov et al (2008) studied the luminescence and radiation effects in electron irradiated $\mathrm{Al}_{2} \mathrm{O}_{3}$ and $\mathrm{Al}_{2} \mathrm{O}_{3}: \mathrm{Cr}$. He observed the emission spectra of both non irradiated and electron irradiated ruby crystals in the red region which show a well known set of narrow emission lines around $700 \mathrm{~nm}$ and attributed the emission due to the transitions from the lowest excited ${ }^{2} E$ state to the ground state ${ }^{4} A_{2}$ of $\mathrm{Cr}^{3+}$. Singh and coworkers (2008) studied the properties of red emitting $\mathrm{Cr}^{3+}$ doped barium aluminate phosphor obtained by combustion process. They reported the PL spectrum with a doublet at $701 \mathrm{~nm}$ and $705 \mathrm{~nm}$. Such a doublet, an intense $R_{1}$ line $(705 \mathrm{~nm})$ and partly frozen out $R_{2}$ line $(701 \mathrm{~nm})$, is the characteristic of $\mathrm{Cr}^{3+}$ ions which are caused by the spin forbidden ${ }^{2} E_{\mathrm{g}} \rightarrow{ }^{4} A_{2 \mathrm{~g}}$ transition. Hence in the present investigations the $R_{1}$ and $R_{2}$ lines are attributed to ${ }^{2} E_{\mathrm{g}} \rightarrow{ }^{4} A_{2 \mathrm{~g}}$ transitions (Nagabhushana 2008).

The PL spectra in the range $600-800 \mathrm{~nm}$ due to $\mathrm{Cr}^{3+}$ ions in pristine and $\mathrm{SHI}$ irradiated one is given as inset in figure 3 . It is observed that, the pristine samples show maximum $\mathrm{PL}$ intensity due to $\mathrm{Cr}^{3+}$ ions whereas it decreases with increase in ion fluence. For the fluence of $1 \times 10^{13}$ ions $\mathrm{cm}^{-2}$ the emission is drastically reduced. The variation of PL intensity of $\mathrm{Cr}^{3+}$ doublet $R_{1}$ and $R_{2}$ lines with ion fluence is given in figure 4 . Disorder due to SHI irradiation can be considered responsible for the reduction in PL intensity. As is widely known (Canham 1997), one of the structural changes causing reduction in $\mathrm{PL}$ intensity is due to defects acting as nonradiative recombination centres whose concentration certainly increases with increasing ion fluence. Further, $\mathrm{Cr}^{3+}$ ions are displaced from octahedrally coordinated oxygen environment due to SHI irradiation which leads to the decrease in volumetric concentration of $\mathrm{Cr}^{3+}$ ions. Therefore, the decrease in intensity of PL at $675 \mathrm{~nm}$ and

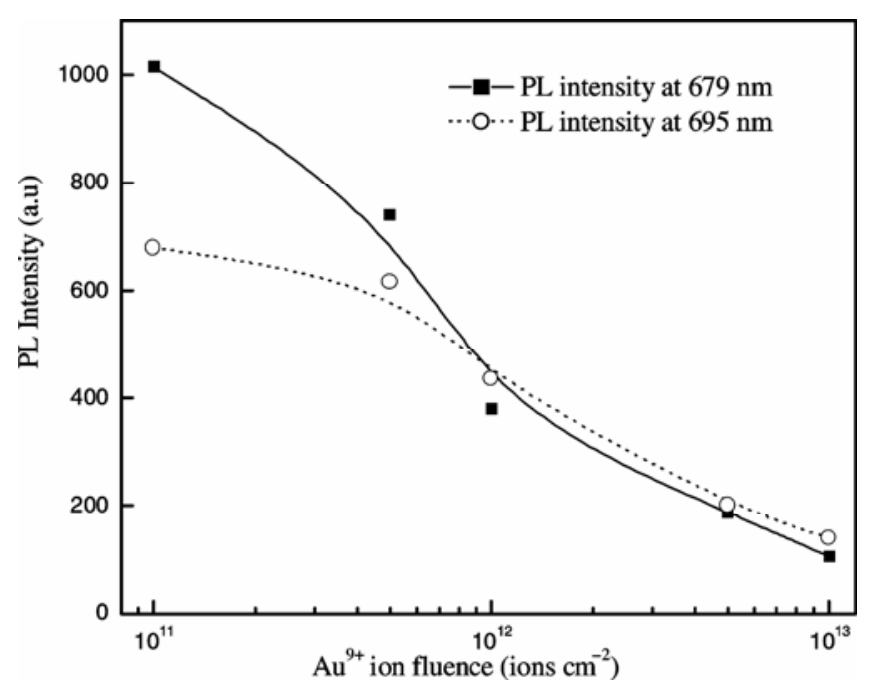

Figure 4. Variation of PL intensity at $R_{1}$ and $R_{2}$ lines of $\mathrm{Cr}^{3+}$ ion emission with irradiation fluence.
$695 \mathrm{~nm}$ is attributed to amorphization. Also, the decrease in PL intensity can be attributed to increase in porosity due to SHI irradiation. Further, SHI irradiation creates a latent track in a target material when Se exceeds a threshold value $\left(S_{\text {eth }}\right)$. Aluminum oxide being a high-band gap insulator, SHI irradiation is expected to create latent tracks in the medium. However, it is reported that the latent tracks were not observed in $\mathrm{Al}_{2} \mathrm{O}_{3}$ (Skuratov et al 2002) using SHI ion beam having $\mathrm{Se}<30 \mathrm{keV} \mathrm{nm}^{-1}$. But, discontinuous tracks can be formed when Se exceeds $18 \mathrm{keV} \mathrm{nm}^{-1}$ (Ramos et al 1998). Therefore, it is believed that, these discontinuous tracks may cause porosity in the sample. At higher fluence the concentration of these discontinuous tracks is higher (Krishna et al 2003). Hence, the PL spectrum is correlated to the surface amorphization due to SHI irradiation.

\section{Conclusions}

Combustion synthesized polycrystalline aluminum oxide shows $\alpha$-phase. GAXRD studies show decrease in the diffraction peak intensity when irradiated with $120 \mathrm{MeV}$ $\mathrm{Au}^{9+}$ ions for a fluence of $1 \times 10^{13}$ ions $\mathrm{cm}^{-2}$. The Raman bands observed at 396, 417, 546, 630 and $842,867 \mathrm{~cm}^{-1}$ have been assigned to $E_{\mathrm{g}}$ (external), $A_{\mathrm{lg}}, E_{\mathrm{g}}$ (external), $A_{\mathrm{gg}}, E_{\mathrm{g}}$ (internal) modes, respectively. The intensity of these bands decreases with increase in ion fluence. This may be attributed to surface amorphization. Further, the PL intensity at 679 and $695 \mathrm{~nm}$ corresponding to $R_{1}$ and $R_{2}$ lines of $\mathrm{Cr}^{3+}$ decreases with increase in ion fluence. This might be due to surface amorphization by discontinuous tracks created by SHI irradiation. Hence, Raman and PL spectroscopic techniques are applied to understand the surface amorphization in combustion synthesized polycrystalline aluminum oxide.

\section{Acknowledgements}

The authors express their sincere thanks to Pelletron group at Inter University Accelerator Centre (IUAC), New Delhi, for proving stable beam during the experiment. This work was supported by grant no 37314 under UFUP scheme of IUAC, New Delhi. One of the authors (KRN) is grateful to the Council of Scientific and Industrial Research (CSIR), for providing a senior research fellowship.

\section{References}

Bolse W, Schattat B, Feyh A and Renz T 2004 Nucl. Instrum. \& Meth. B218 80

Brundle C R, Evans Jr C K and Wilson S 1992 Encyclopedia of materials characterization (Butterworth Heinemann) p. 437

Canham L (ed.) 1997 Properties of porous silicon (London: INSPEC) 
Jankowiak R, Roberts K, Tomasik P, Sikora M, Small G J and Schilling C H 2000 Mater. Sci. Eng. A281 45

Kadleikova M, Breza J and Vesely M 2001 Microelectronics J. 32955

Kingsley J J and Pail K C 1988 Mater. Lett. 6427

Krishna R, Kessavamoorthy R, Dash S, Tyagi A K and Raj B 2003 Scr. Mater. 481099

Krishnan R S 1947 Proc. Indian Acad. Sci. A26 450

Makhov V N, Lushchik A, Lushchik Ch B, Kirm M, Vasil'chenko E, Vielhauer S, Harutunyan V V and Aleksanyan E 2008 Nucl. Instrum. \& Meth. 2662949

McKeever S W S, Akselrod M S, Colyott L E, Larsen N A, Polf J C and Whitley V 1999 Radiat. Protect. Dosim. 84163

Mimani T 2000 Resonance 550

Nagabhushana K R 2008 Ph D thesis, Bangalore University, Bangalore

Nagabhushana H, Lakshminarasappa B N, Prashantha S C, Nagabhushana K R and Fouran Singh 2006 Nucl. Instrum. \& Meth. B244 31
Nagabhushana H, Prashantha S C, Nagabhushana B M, Lakshminarasappa B N and Fouran Singh 2008 Spectrochim. Acta A71 1070

Porto S P S and Krishnan R S 1967 J. Chem. Phys. 47 1009

Ramos S M M, Banardi N, Canut B, Bouffard S and DellaNegra S 1998 Nucl. Instrum. Meth. B143 319

Ryazanov A I and Kinoshita C 2002 Nucl. Instrum. Meth. B191 65

Skuratov V A, Efimov A E and Zagorskii D L 2002 Phys. Solid State $\mathbf{4 4} 171$

Song Y, Zhang C H, Wang Z G, Sun Y M, Daun J L and Zhao Z M 2006 Nucl. Instrum. \& Meth. B245 120

Singh Vijay, Chakradhar R P S, Rao J L and Jun-Jie Zhu 2008 Mater. Chem. \& Phys. 111143

Wang Z G, Zhao Z M, Song Y, Liu J, Sun Y M, Zhang C H, Daun J L and Lin Y F 2004 Nucl. Instrum. \& Meth. B218 472

Ziegler J F 2004 Nucl. Instrum. \& Meth. 219-220 1027 